\title{
3 Research Square

\section{Circ_0038467 is Overexpressed in Osteoarthritis and it Promotes the Maturation of miR-203 to Increase the Apoptosis of Chondrocytes Induced by LPS}

\section{Zhongkun Gou}

Shenzhen Baoan Shiyan People's Hospital

Quanling Wu

Shenzhen Baoan Shiyan People's Hospital

Changqing Jiang

Shenzhen Baoan Shiyan People's Hospital

Wei Dong ( $\sim$ WeiDongBone@163.com )

Shenzhen Baoan Shiyan People's Hospital

Research

Keywords: Circ_0038467, osteoarthritis, miR-203, chondrocytes, maturation

Posted Date: January 28th, 2021

DOl: https://doi.org/10.21203/rs.3.rs-153996/v1

License: (c) (1) This work is licensed under a Creative Commons Attribution 4.0 International License.

Read Full License 


\section{Abstract}

Introduction: LPS-induced inflammation contributes to osteoarthritis (OA). It is known that Circ_0038467 plays a critical role in LPS-mediated inflammation, suggesting the its involvement in OA. This study aimed to study the possible involvement of Circ_0038467 in OA.

Materials and Methods: Circ_0038467, mature miR-203 and miR-203 precursor expression in controls and OA patients was determined by RT-qPCR. Circ_0038467 overexpression in chondrocytes and RT-qPCR was performed to analyze its effects on the expression of mature miR-203 and miR-203 precursor. The role of Circ_0038467 and miR-203 in cell apoptosis was analyzed by cell apoptosis assay.

Results: Circ_0038467 was upregulated in OA and positively correlated with mature miR-203, but not miR203 precursor. In chondrocytes, increased expression of both Circ_0038467 and miR-203 was observed after LPS treatment. In chondrocytes, Circ_0038467 overexpression increased the expression levels of mature miR-203, but not miR-203 precursor. Analysis of cell apoptosis showed that overexpression of Circ_0038467 and miR-203 increased cell apoptosis. In addition, miR-203 inhibitor reversed the effects of Circ_0038467 overexpression on cell apoptosis.

Conclusions: Circ_0038467 is highly expressed in OA and may promote the production of mature miR203 to increase the apoptosis of chondrocytes induced by LPS.

\section{Introduction}

With aging, the loss and degradation of the articular cartilage will occur, leading to the development of osteoarthritis (OA), which is a type of chronic degenerative disease (1). Among the population older than 60 years, more than $13 \%$ of females and $10 \%$ of males are suffering from OA (2). Despite the advances in the diagnosis and treatment of OA, there is still no cure for OA (3). Current available treatment approaches mainly focus on symptom relief (4). Even worse, these treatments may not work on severe $\mathrm{OA}(3,4)$. To this end, novel therapeutic approaches are needed to improve the quality of life of OA patients. However, molecular pathogenesis of OA remains hardly known, which limits the discovery of novel treatments $(5,6)$.

Molecular factors play important roles in the development and progression of OA $(7,8)$. Some pathways, such as Notch pathway, NF-KB pathway and PCP/JNK-mTORC1-PTHrP cascade, have been proven to be critical players in $\mathrm{OA}(7,8)$. In effect, some molecular factors are promising targets for targeted $\mathrm{OA}$ patients $(9,10)$. However, safe and effective targets for OA remain to be developed. CcircRNAs are closed (covalently) RNA transcripts with critical functions in gene expression regulation rather than coding protein $(11,12)$. In OA, CircRNAs not only participates in disease progression, but also serves as biomarkers for disease diagnosis and prognosis(13), suggesting the potential roles of circRNAs as promising targets for OA targeted therapy. The development of osteoarthritis (OA) requires the involvement of LPS-induced inflammation (14), in which circRNA Circ_0038467 plays a critical role (15), suggesting the involvement of Circ_0038467 in OA. Our deep sequence analysis revealed the altered 
expression of Circ_0038467 and its inverse correlation with miR-203 (data not shown), which is also a critical player in LPS-induced cell injury(16). We therefore analyze the potential interaction between Circ_0038467 and miR-203 in OA.

\section{Materials And Methods Participants}

We in this study enrolled a total of 60 controls (20 males and 40 females) and 60 OA patients (hip OA, 20 males and 40 females) at Shenzhen Baoan Shiyan People's Hospital between June 2018 and June 2020. Ethics Committee of this hospital approved this study. Patients with a history of OA or the ones with initiated therapy were excluded from this study. Patients complicated multiple clinical disorders were also not included. Healthy controls were enrolled at the physiological health center. The physiological functions of all organs of the healthy controls were within normal range. Age range of both PA patients and healthy controls was 56 to 70 years, with a median of 63 years. All patients and healthy controls signed informed consent.

\section{Synovial fluid, treatment and chondrocytes derived from an OA patients}

Under local anesthesia, a syringe was used to extract synovial fluid ( 1.5 to $2.0 \mathrm{ml}$ ) from the affected hip of OA patients. To serve as controls, similar amount of synovial fluid was also extracted from the hip of healthy controls. All patients were treated with NSAIDs. NSAIDs exert their anti-inflammatory and analgesic effects to alleviate OA by inhibiting the prostaglandin-generating enzyme, cyclooxygenase. At 1 month after treatment, symptoms were improved in all cases. At this time point, synovial fluid was also extract from OA patients. Synovial fluid samples were kept in liquid nitrogen storage prior to the subsequent assays.

OA-affected chondrocytes (4020A-05A, Sigma-Aldrich) were used. Chondrocyte growth medium purchased from PromoCell was used to cultivate cells in an incubator at $5 \% \mathrm{CO}_{2}, 95 \%$ humidity and $37^{\circ} \mathrm{C}$. To study the effects of LPS treatment on gene expression, chondrocytes were cultivated in medium containing 0, 2, 4, 8 and $10 \mu \mathrm{g} / \mathrm{ml}$ LPS (Sigma-Aldrich) for $48 \mathrm{~h}$ prior to the subsequent assays.

\section{Cell transfections}

Expression vector of Circ_0038467 was established using pcDNA3.1(+) CircRNA Mini Vector (Addgene) as backbone. Mimic of miR-203 and miRNA precursor as well as miR-203 inhibitor and NC inhibitor were designed and synthesized by Invitrogen (Shanghai, China). Lipofectamine 2000 (Invitrogen) was used to transfect $1 \mu \mathrm{g}$ Circ_0038467 expression vector, 40 nM miR-203 inhibitor, or 40 nM miR-203 into 
chondrocytes. Control (untransfected cells, C) and NC (NC miRNA-, NC inhibitor- or empty vectortransfected cells) experiments were also included.

\section{RNA isolation and genomic DNA removal}

Ribozol (VMR) was used to extract total RNA from both synovial fluid and chondrocytes, followed by digesting genomic DNA with DNase I (Invitrogen) for $2 \mathrm{~h}$ at $37^{\circ} \mathrm{C}$. Electrophoresis performed using ureaPAGE gels (5\%) was used to analyze RNA integrity. RNA purity was analyzed by measuring the OD260/280 ratios.

\section{RT-qPCRs}

Only RNA samples with an OD260/280 ratio close to 2.0 were used as template to prepare cDNA samples. To determine the expression of Circ_0038467, qPCRs were performed using SYBR Green Master Mix (Bio-Rad) with GAPDH as an internal control. To determine the expression of miR-203 precursor, the same method was used to perform RTs and qPCRs with sequence-specific primers.

To determine the expression of mature miR-203, All-in-One ${ }^{T M}$ miRNA qRT-PCR Detection Kit* (GeneCopoeia) was used to perform poly $(A)$ addition, followed by using poly $(T)$ as reverse primer to carry out RTs and qPCRs. U6 was used as the internal control.

Primer sequences were: circ_0038467: 5'-TCCCAGCTGACCTAAAGTCAAT-3' (forward) and 5'TGGTGACATTGAGCAGGAAC-3' (reverse), GAPDH: 5'-GTCAGCCGCATCTTCTTTTG-3' (forward) and 5'GCGCCCAATACGACCAAATC-3' (reverse); miR-203 precursor: 5'-TGTGTTGGGGACTCGCGCGCGT-3' (forward) and 5'-TCGCTGTCGCCGCGCGCC-3' (reverse); mature miR-203:5'-

AGTGGTTCTTAACAGTTCAAC-3' (forward) and poly (T). U6 primers were from the kit.

$\mathrm{Ct}$ values of target genes were normalized to internal controls using $2^{-\Delta \Delta \mathrm{Ct}}$ method. The sample with the biggest $\Delta \mathrm{Ct}$ was set to value " 1 ", all other samples were normalized to this sample.

\section{Apoptosis assay}

Apoptosis of chondrocytes was analyzed at $48 \mathrm{~h}$ post-transfection. In brief, chondrocytes were washed with PBS and cultivated in medium supplemented with $10 \mu \mathrm{g} / \mathrm{ml}$ LPS for $48 \mathrm{~h}$. After that, chondrocytes were washed with pre-cold PBS and staining with Annexin-V FITC and propidium iodide (PI) for 12 min in dark was followed. Finally, FACSCalibur instrument was used to analyze cell apoptosis.

\section{Statistical analysis}


Unpaired t test was used to compare $\mathrm{OA}$ and control group. Data of multiple independent groups were analyzed by ANOVA Tukey's test. $\mathrm{P}<0.05$ was statistically significant.

\section{Results}

\section{Circ_0038467 was upregulated in OA and was downregulated after treatment}

Our RT-qPCR data showed that Circ_0038467 was upregulated in OA compared to control group (Fig.1A, $p<0.01)$. All patients were treated with NSAIDs. At 1 month after treatment, symptoms were improved in all cases. Circ_0038467 expression in synovial fluid samples from OA patients was also determined by RT-qPCR at this time point. It was observed that Circ_0038467 expression was decreased after treatment (post-treatment) compared to pre-treatment level (Fig.1B, $p<0.01$, paired t test).

\section{Mature miR-203 and miR-203 precursor were upregulate in OA but only mature miR-203 was positively correlated with Circ_0038467}

Our RT-qPCR data showed that mature miR-203 (Fig.1A) and miR-203 precursor (Fig.1B) was upregulated in OA compared to control group $(p<0.01)$. Pearson's correlation coefficient analysis showed that Circ_0038467 expression was positively correlated with mature miR-203 (Fig.2C), but not miR-203 precursor (Fig.2C) across synovial fluid samples from OA patients.

\section{Circ_0038467 overexpression upregulated the expression of mature miR-203 in chondrocytes}

Confirmation of Circ_0038467 and miR-203 overexpression was performed every $24 \mathrm{~h}$ until $96 \mathrm{~h}$. It was observed that Circ_0038467 and miR-203 were significantly overexpressed between 48h and 96h (Fig.3A, $\mathrm{p}$ <0.05). It was observed that Circ_0038467 overexpression increased the expression of mature miR-203 between $48 \mathrm{~h}$ and $96 \mathrm{~h}$ (Fig.3B, p<0.05), but failed to significantly affect the expression of miR-203 precursor at each time point (Fig.3C). Moreover, overexpression of miR-203 failed to significantly affect the expression of Circ_0038467 at each time point (Fig.3D).

\section{Circ_0038467 overexpression increased LPS-induced apoptosis of chondrocytes through miR-203}

To study the effects of LPS treatment on the expression Circ_0038467 and miR-203, chondrocytes were cultivated in medium with LPS for 48h, followed by the determination of the expression of Circ_0038467 
(Fig.4A) and miR-203 (Fig.4B) by RT-qPCR. LPS increased the expression of Circ_0038467 and miR-203 in a dose-dependent manner $(\mathrm{p}<0.05)$. Analysis of the apoptosis of chondrocytes induced by LPS showed that overexpression of Circ_0038467 and miR-203 increased cell apoptosis. In addition, miR-203 inhibitor reversed the effects of Circ_0038467 overexpression on cell apoptosis (Fig.4C, $p<0.05$ ).

\section{Discussion}

In this study, the differential expression of Circ_0038467 and miR-203 in OA, as well as the potential interactions between them were explored. We showed that both Circ_0038467 and miR-203 were upregulated in OA. Interestingly, Circ_0038467 could promote the maturation of miR-203 to promote the apoptosis of chondrocytes induced by LPS.

Liu et al. reported that Circ_0038467 was upregulated in human bronchial epithelial cells by LPS treatment and the knockdown of Circ_0038467 protected cells from LPS-induced injury (15), suggesting that Circ_0038467 may promote LPS-induce cell injury. Consistently, our study also showed that LPS treatment increased the expression of Circ_0038467 in chondrocytes in a dose-dependent manner. Chondrocytes are the only mature cells found in cartilage (17). The main function of chondrocytes is to maintain and sustain the cartilage by secreting extracellular matrix (17). During OA, apoptosis of chondrocytes will be increased, leading to the degradation of cartilage matrix (17). We showed that Circ_0038467 overexpression increased the apoptosis of chondrocytes under LPS treatment. In addition, Circ_0038467 was also overexpressed in OA patients compared to healthy controls. Therefore, LPSinducible Circ_0038467 may promote OA by increasing the apoptosis of chondrocytes.

MiR-203 knockdown can target MCL-1 to reduce LPS-induced injury in chondrocytes (16), suggesting the involvement of miR-203 in OA. In this study we showed that miR-203 was overexpressed in OA at both precursor and mature miRNA levels. Moreover, miR-203 is also LPS-inducible, and overexpression of miR203 increased the apoptosis of chondrocytes under LPS treatment. Therefore, miR-203 may also promote OA progression by increasing LPS-induced apoptosis of chondrocytes.

Interestingly, we found that Circ_0038467 overexpression increased the expression of mature miR-203, but not miR-203 precursor in chondrocytes. Therefore, Circ_0038467 may suppress the maturation of miR-203. Considering the fact that the maturation of miRNAs requires the movement of miRNA precursor from nucleus to cytoplasm, Circ_0038467 may promote the transportation of miR-203 precursor to increase its maturation. Our future studies will explore this possibility.

\section{Conclusion}

In conclusion, Circ_0038467 and miR-203 are overexpressed in OA. In addition, Circ_0038467 may promote the maturation of miR-203 to increase the apoptosis of chondrocytes induced by LPS, thereby promoting the progression of $\mathrm{OA}$. 


\section{Declarations}

\section{Ethical Approval and Consent to participate}

Informed consent was obtained from all individual participants included in the study. All producers were approved by Shenzhen Baoan Shiyan People's Hospital Ethics Committee. Procedures operated in this research were completed in keeping with the standards set out in the Announcement of Helsinki and laboratory guidelines of research in China.

\section{Consent to publish}

Authors are responsible for correctness of the statements provided in the manuscript. See also Authorship Principles. The Editor-in-Chief reserves the right to reject submissions that do not meet the guidelines described in this section.

\section{Availability of supporting data}

The data that support the findings of this study are available on request from the corresponding author: * Wei Dong, Department of Bone and Joint Surgery, Shenzhen Baoan Shiyan People's Hospital, No.11 Jixiang Road, Shiyan Street, Baoan District, Shenzhen City, Guangdong Province, 518108, PR. China. Email address: WeiDongBone@163.com.

The data are not publicly available due to their containing information that could compromise the privacy of research participants.

\section{Competing interests}

All other authors have no conflicts of interest.

We declare that we do not have any commercial or associative interest that represents a conflict of interest in connection with the work submitted.

\section{Funding}

Not Applicable.

\section{Authors' contributions}


Zhongkun Gou, Wei Dong: study concepts, literature research, clinical studies, data analysis, experimental studies, manuscript writing and review; Quanling Wu: study design, literature research, experimental studies and manuscript editing; Changqing Jiang: definition of intellectual content, clinical studies, data acquisition and statistical analysis.

The authors declare that all data were generated in-house and that no paper mill was used.

\section{Acknowledgements}

Not Applicable.

\section{References}

1. Glyn-Jones S, Palmer AJ, Agricola R, Price AJ, Vincent TL, Weinans H, et al. Osteoarthritis. Lancet. 2015;386(9991):376-87.

2. Zhang Y, Jordan JM. Epidemiology of osteoarthritis. Clinics in geriatric medicine. 2010;26(3):355-69.

3. Sinusas K. Osteoarthritis: diagnosis and treatment. American family physician. 2012;85(1):49-56.

4. Hügle T. [Update Osteoarthritis]. Revue medicale suisse. 2020;16(685):500-2.

5. Charlier E, Relic B, Deroyer C, Malaise O, Neuville S, Collée J, et al. Insights on Molecular Mechanisms of Chondrocytes Death in Osteoarthritis. International journal of molecular sciences. 2016;17(12).

6. Chen D, Shen J, Zhao W, Wang T, Han L, Hamilton JL, et al. Osteoarthritis: toward a comprehensive understanding of pathological mechanism. Bone research. 2017;5:16044.

7. Saito T, Tanaka S. Molecular mechanisms underlying osteoarthritis development: Notch and NF-KB. Arthritis research \& therapy. 2017;19(1):94.

8. Tong W, Zeng Y, Chow DHK, Yeung W, Xu J, Deng Y, et al. Wnt16 attenuates osteoarthritis progression through a PCP/JNK-mTORC1-PTHrP cascade. Annals of the rheumatic diseases. 2019;78(4):551-61.

9. Carpintero-Fernandez P, Varela-Eirin M, Lacetera A, Gago-Fuentes R, Fonseca E. New Therapeutic Strategies for Osteoarthritis by Targeting Sialic Acid Receptors. 2020;10(4).

10. Conaghan PG, Cook AD, Hamilton JA, Tak PP. Therapeutic options for targeting inflammatory osteoarthritis pain. Nature reviews Rheumatology. 2019;15(6):355-63.

11. Greene J, Baird AM, Brady L, Lim M, Gray SG, McDermott R, et al. Circular RNAs: Biogenesis, Function and Role in Human Diseases. Frontiers in molecular biosciences. 2017;4:38.

12. Haque S, Harries LW. Circular RNAs (circRNAs) in Health and Disease. Genes. 2017;8(12).

13. Li HZ, Lin Z, Xu XH, Lin N, Lu HD. The potential roles of circRNAs in osteoarthritis: a coming journey to find a treasure. Bioscience reports. 2018;38(5).

14. Huang ZY, Stabler T, Pei FX, Kraus VB. Both systemic and local lipopolysaccharide (LPS) burden are associated with knee OA severity and inflammation. Osteoarthritis and cartilage. 2016;24(10):176975 . 
15. Liu G, Wan Q, Li J, Hu X, Gu X, Xu S. Circ_0038467 regulates lipopolysaccharide-induced inflammatory injury in human bronchial epithelial cells through sponging miR-338-3p. 2020;11(5):1297-308.

16. Zhao C, Wang Y, Jin H, Yu T. Knockdown of microRNA-203 alleviates LPS-induced injury by targeting MCL-1 in C28/I2 chondrocytes. Experimental cell research. 2017;359(1):171-8.

17. Hwang HS, Kim HA. Chondrocyte Apoptosis in the Pathogenesis of Osteoarthritis. International journal of molecular sciences. 2015;16(11):26035-54.

\section{Figures}

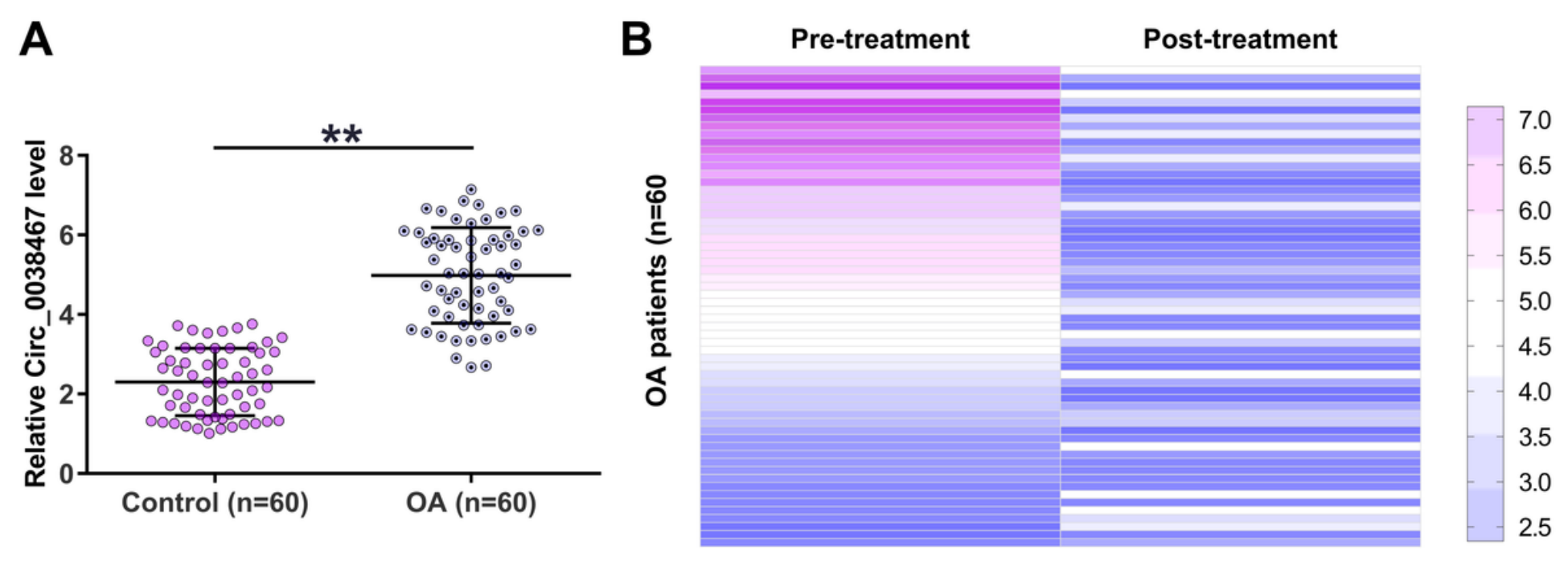

\section{Figure 1}

Circ_0038467 was upregulated in OA and was downregulated after treatment Circ_0038467 expression was studied by RT-qPCR. Unpaired $t$ test was used to compare two groups $(A) .{ }^{*}, p<0.01$. All patients were treated with NSAIDs. Circ_0038467 expression in synovial fluid samples from OA patients was also determined by RT-qPCR at 1 month after treatment. Heatmaps were plotted using Heml 1.0 software to reflect changes in gene expression levels during treatment. 

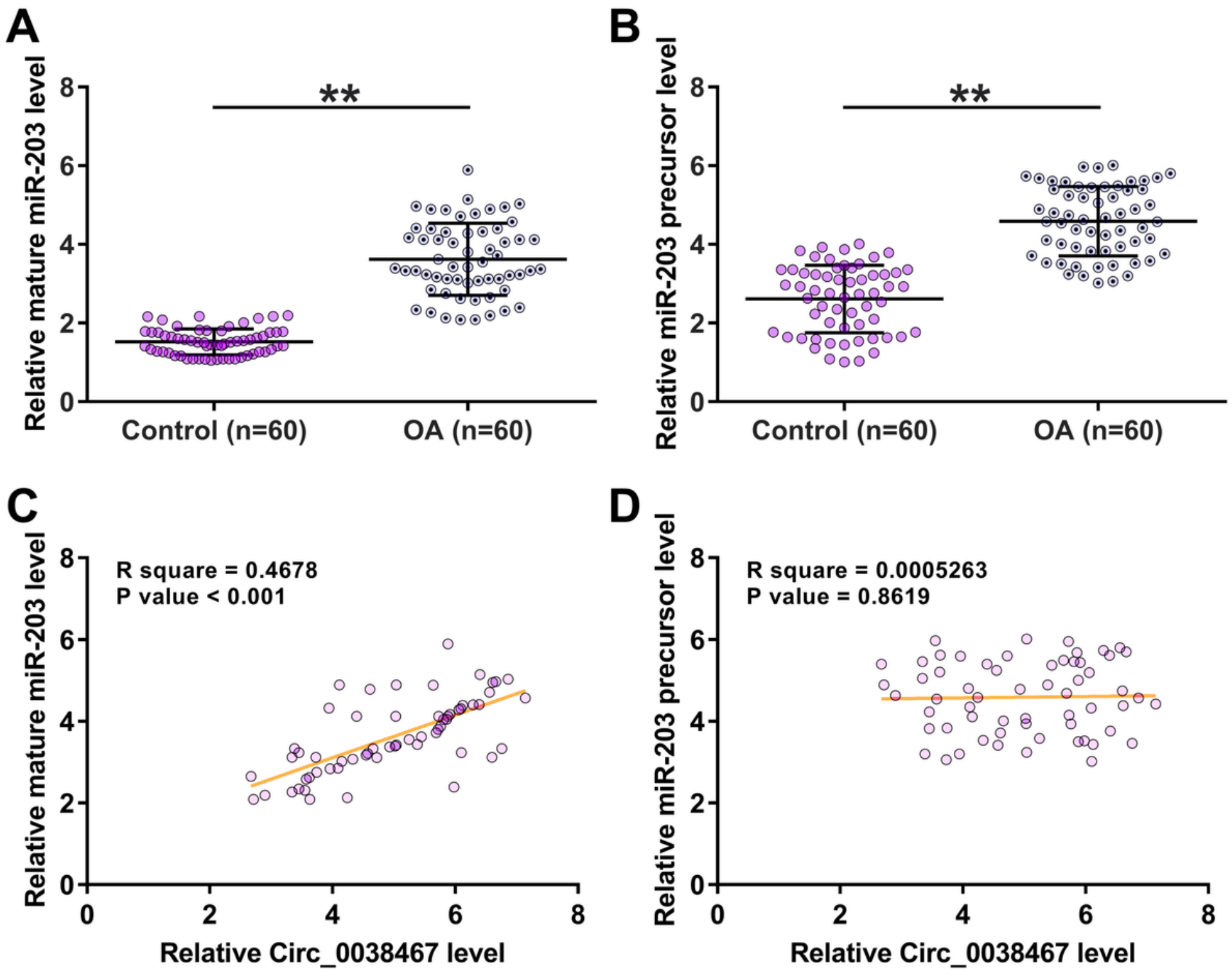

Figure 2

Mature miR-203 and miR-203 precursor were upregulate in OA but only mature miR-203 was positively correlated with Circ_0038467 Mature miR-203 and miR-203 precursor expression was studied by RTqPCR. Unpaired t test was used to compare the expression levels of mature miR-203 (A) and miR-203 precursor (B) between two groups $(A) .{ }^{\star \star}, p<0.01$. Pearson's correlation coefficient analysis was performed to analyze the correlations between Circ_0038467 and mature miR-203 (C) or miR-203 precursor (D) across synovial fluid samples from OA patients. 

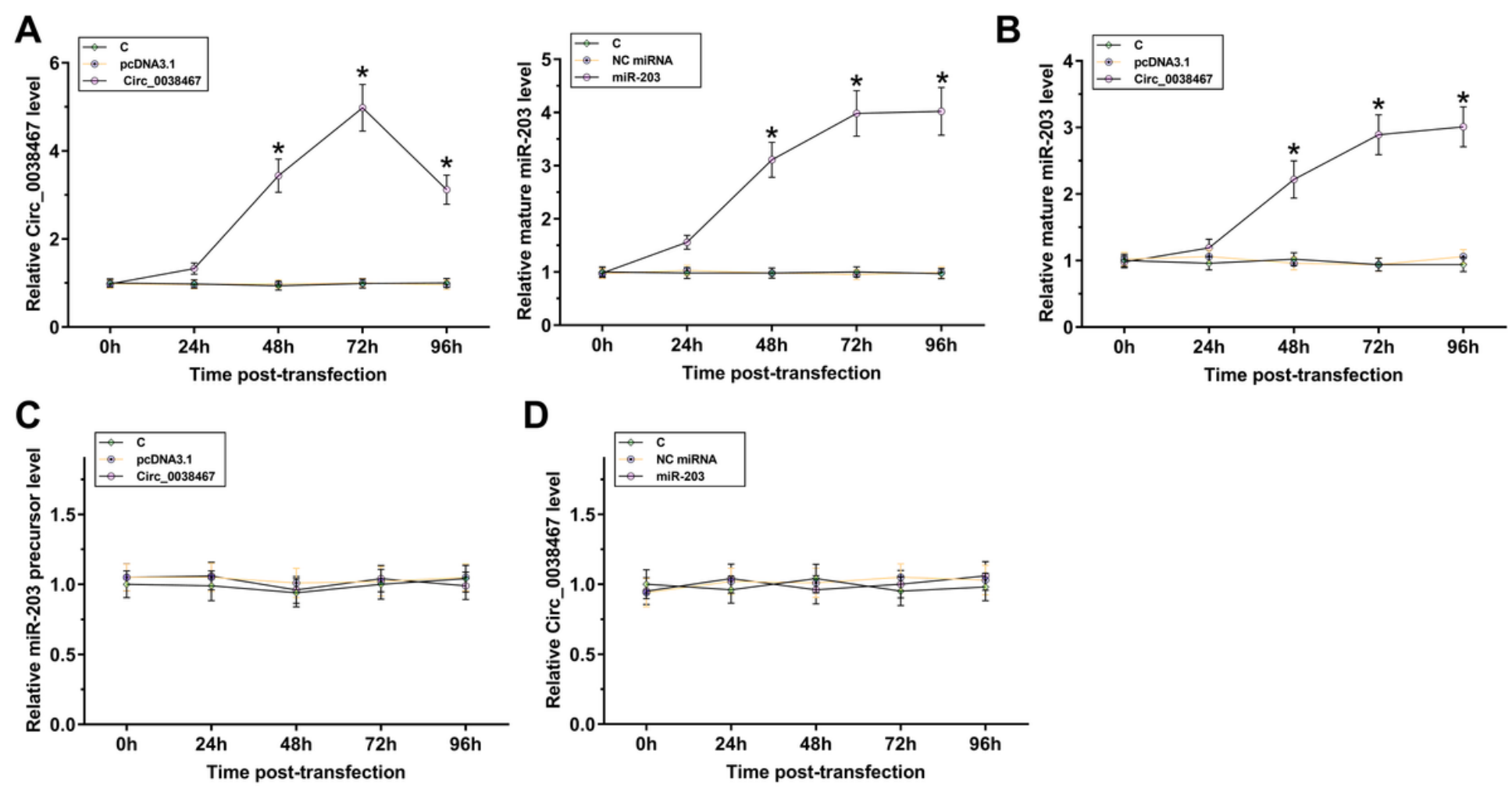

\section{Figure 3}

Circ_0038467 overexpression upregulated the expression of mature miR-203 in chondrocytes Circ_0038467 expression vector or miR-203 mimic was transfected into chondrocytes, followed by the confirmation of Circ_0038467 (A) and miR-203 (B) overexpression every 24h until 96h. The effects of Circ_0038467 overexpression on the expression of mature miR-203 (B) and miR-203 precursor (C), and the effects of miR-203 overexpression on Circ_0038467 (D) were analyzed by RT-qPCR at each time point. ${ }^{*}, \mathrm{p}<0.05$. 


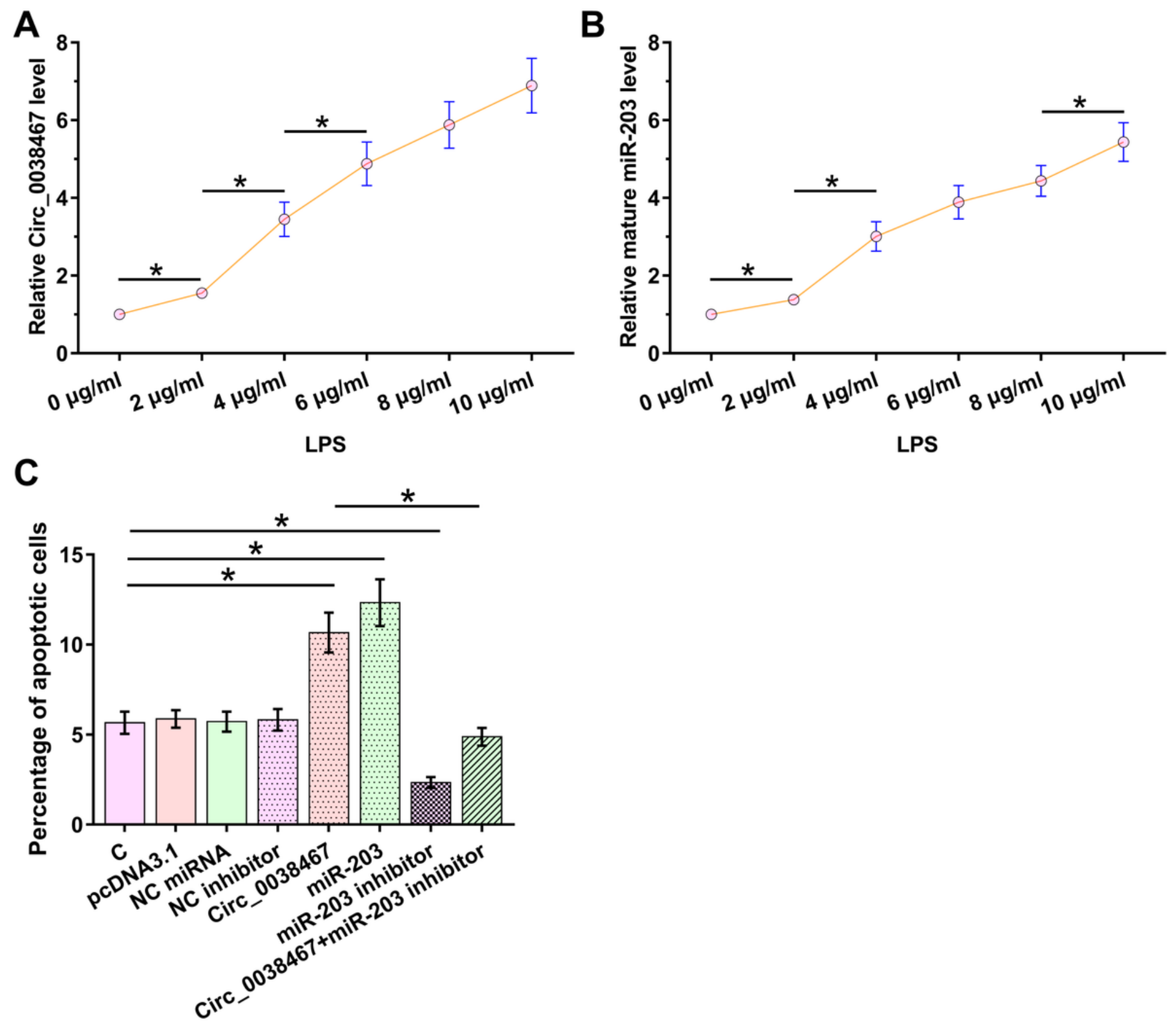

Figure 4

Circ_0038467 overexpression increased LPS-induced apoptosis of chondrocytes through miR-203 To study the effects of LPS treatment on the expression Circ_0038467 and miR-203, chondrocytes were cultivated in medium containing $0,2,4,8$ and $10 \mu \mathrm{g} / \mathrm{ml}$ LPS for $48 \mathrm{~h}$, followed by the determination of the expression of Circ_0038467 (A) and miR-203 (B) by RT-qPCR. The role of Circ_0038467 and miR-203 in regulating the apoptosis of chondrocytes was analyzed by cell apoptosis assay (C). ${ }^{*}, \mathrm{p}<0.05$. 